\title{
ARTICLE
}

\section{Transcriptome analyses of the Dof-like gene family in grapevine reveal its involvement in berry, flower and seed development}

\author{
Danielle Costenaro da Silva ${ }^{1,6,7}$, Vítor da Silveira Falavigna ${ }^{1,6}$, Marianna Fasoli ${ }^{2,8}$, Vanessa Buffon ${ }^{3}$, Diogo Denardi Porto ${ }^{4}$, \\ Georgios Joannis Pappas $\mathrm{Jr}^{5}$, Mario Pezzotti ${ }^{2}$, Giancarlo Pasquali ${ }^{1}$ and Luís Fernando Revers ${ }^{3}$
}

The Dof (DNA-binding with one finger) protein family spans a group of plant transcription factors involved in the regulation of several functions, such as plant responses to stress, hormones and light, phytochrome signaling and seed germination. Here we describe the Dof-like gene family in grapevine (Vitis vinifera L.), which consists of 25 genes coding for Dof. An extensive in silico characterization of the $V v i D o f L$ gene family was performed. Additionally, the expression of the entire gene family was assessed in 54 grapevine tissues and organs using an integrated approach with microarray (cv Corvina) and real-time PCR (cv Pinot Noir) analyses. The phylogenetic analysis comparing grapevine sequences with those of Arabidopsis, tomato, poplar and already described Dof genes in other species allowed us to identify several duplicated genes. The diversification of grapevine DofL genes during evolution likely resulted in a broader range of biological roles. Furthermore, distinct expression patterns were identified between samples analyzed, corroborating such hypothesis. Our expression results indicate that several VviDofL genes perform their functional roles mainly during flower, berry and seed development, highlighting their importance for grapevine growth and production. The identification of similar expression profiles between both approaches strongly suggests that these genes have important regulatory roles that are evolutionally conserved between grapevine cvs Corvina and Pinot Noir.

Horticulture Research (2016) 3, 16042; doi:10.1038/hortres.2016.42; Published online 31 August 2016

\section{INTRODUCTION}

Dof (DNA-binding with one finger) proteins are plant exclusive transcription factors characterized by a conserved 52 amino acid segment encompassing a $\mathrm{C}_{2} \mathrm{C}_{2}$-type zinc finger. ${ }^{1}$ The Dof domain is usually found in the $\mathrm{N}$-terminal region of these proteins, specifically binding AAAG sequences in gene promoters. The C-terminal region is responsible to interact with other regulatory proteins in order to activate gene expression (reviewed in Yanagisawa ${ }^{1}$ and Hir and Bellini ${ }^{2}$ ). In contrast to the highly conserved DNA-binding domain, sequences outside the Dof domain diverge widely, suggesting that Dof proteins are regulating several biological functions. Indeed, functional studies conducted so far confirm this assumption, demonstrating Dof involvement in seed germination, ${ }^{3-9}$ seed development, ${ }^{10-14}$ flowering control, ${ }^{15-18}$ flower abscission, ${ }^{19}$ pollen development, ${ }^{20}$ among many other functions (recently reviewed in Gupta et al. ${ }^{21}{ }^{2} \mathrm{Hir}$ and Bellini ${ }^{2}$ and Noguero et al. ${ }^{22}$ ).

After the first isolation of a complementary DNA from maize encoding a Dof domain-containing protein, these transcription factors have been identified in a variety of angiosperms including Arabidopsis, barley, pea, potato, pumpkin, rice, tobacco and wheat. ${ }^{1}$ Thereafter, Dof-like genes were identified in several other species such as grapevine, ${ }^{23}$ Chlamydomonas reinhardtii, Jatropha curcas, maritime pine, pea, the moss Physcomitrella patens, sorghum, soybean and sweet potato. ${ }^{21,22}$ In terms of family composition, the first genome-wide report for Dof genes was for Arabidopsis, which presented 36 Dof-encoding genes and one pseudogene. ${ }^{1}$ Thereafter, many studies identifying Dof genes have been reported, with 30 members in rice, ${ }^{24} 41$ in poplar, ${ }^{25} 26$ in barley, ${ }^{26} 19$ in moss, ${ }^{27} 12$ in the fern Selaginella moellendorffiii, ${ }^{27} 10$ in loblolly pine, ${ }^{27} 31$ in wheat, $^{28} 27$ in Brachypodium distachyon, ${ }^{29} 10$ in pine, ${ }^{30} 34$ in tomato, ${ }^{31} 78$ in soybean, ${ }^{32} 25$ in sugarcane, 33 in Chinese cabbage, $^{34} 46$ in maize, ${ }^{35} 35$ in potato ${ }^{36}$ and 46 in carrot. $^{37}$

We have previously conducted a representational difference analysis aiming to identify genes related to seedlessness during specific developmental stages in grapevine. ${ }^{23}$ Among the expressed sequence tags identified, a putative Dof gene was isolated in the developmental stage of four-weeks after fruit set. We therefore decided to further investigate the presence of other genes in the grapevine genome potentially encoding Dof proteins. Here we demonstrate that 25 Dof-like genes are present in grapevine (VviDofL). In silico analyses, including a phylogenetic approach that revealed clusters of paralogous and orthologous genes, allowed the prediction of putative functions for these genes. Finally, the expression profiles of all $25 \mathrm{VviDofL}$ genes were established by whole-transcriptome data and strongly suggested

\footnotetext{
${ }^{1}$ Graduate Program in Cell and Molecular Biology, Center for Biotechnology, Universidade Federal do Rio Grande do Sul, Porto Alegre, RS 91501-970, Brazil; ${ }^{2}$ Dipartimento di Biotecnologie, Università degli Studi di Verona, Verona 37134, Italy; ${ }^{3}$ Embrapa Uva e Vinho, Bento Gonçalves, RS 95701-008, Brazil; ${ }^{4}$ Embrapa Semiárido, Petrolina, PE 56302-970, Brazil and ${ }^{5}$ Department of Cell Biology, Universidade de Brasília, Brasília, DF 70910-900, Brazil.

Correspondence: LF Revers (luis.revers@embrapa.br)

${ }^{6}$ These authors contributed equally to this work.

${ }^{7}$ Present address: Syngenta Seeds Ltda, Uberlândia, MG, 38407-049, Brazil.

${ }^{8}$ Present address: E. \& J. Gallo Winery, Modesto, CA 95354, USA.
}

Received: 30 June 2016; Revised: 8 August 2016; Accepted: 9 August 2016 
the involvement of some of these genes in the regulation of berry, flower and seed development.

\section{MATERIAL AND METHODS}

Bioinformatic analysis of nucleotide and amino acid sequences A VviDofL sequence previously identified by the group ${ }^{23}$ was used as query in a genome-wide search for putative Dofs using the BLASTP tool ${ }^{38}$ implemented in the $12 \mathrm{X}$ Grape Genome Browser V1 (http://genomes.cribi.unipd.it/), ${ }^{39,40}$ which was verified in the V2 version with the same $25 \mathrm{VviDofL}$. In order to confirm the results, the Dof-seed sequence (accession number PF02701) available in the Pfam database ${ }^{41}$ was used to perform a HMM search using Hmmer v3.0 (http://hmmer.org/) to find predicted grapevine proteins in the 12X Grape Genome Browser V1. All predicted gene models identified by both strategies were compared with the Pfam database ${ }^{41}$ in order to confirm the presence of the Dof domain (PF02701), and the ones without the characteristic domain of the gene family were excluded from further analyses. The chromosomal locations of VviDofL genes were obtained at the Grape Genome Browser, whereas marker locations were obtained at the National Center for Biotechnology Information database (http://www.ncbi.nlm.nih.gov/). All data were compiled into a figure using the MapChart v.2.2 (Wageningen, Netherlands) software. ${ }^{42}$

Deduced amino acid sequences of all grapevine DofL proteins were searched for the presence of conserved domains using MEME Suite v.4.9.0. ${ }^{43}$ Default parameters were used with the following exceptions: the motif distribution among sequences was set to any number of repetitions; the maximum number of motifs was set to 15; the maximum motif width was defined between 10 and 52 amino acids; and an e-value cutoff of $1^{-10}$ was adopted. All identified motifs were annotated by comparison with conserved motifs in Pfam $^{41}$ and SMART ${ }^{44}$ databases. Protein subcellular localization was predicted using the Plant-mPLoc database (http:// www.csbio.sjtu.edu.cn/bioinf/plant-multi/\#). ${ }^{45}$ Full-length protein sequences of grapevine, Arabidopsis, poplar, tomato and other already described Dof-encoding genes (searched in March, 2016) were aligned using the ClustalW software. ${ }^{46}$ Accession numbers are shown in Supplementary Table S1. Two phylogenetic trees were inferred using MrBayes v.3.2.6 software $^{47}$ employing the mixed amino acid substitution model in the default settings. The first tree performed a 14.81 million generation run, sampled every 100 generations and the first $25 \%$ trees were discarded as burn-in. The remaining ones were summarized in a consensus tree. The alignment of the second tree was cured to eliminate poor alignment positions and divergent regions using Gblocks $0.91 \mathrm{~b}$ (Barcelona, Spain) software in default settings, except gap positions that were set to half. ${ }^{48}$ The cured alignment was composed of 50 amino acids spanning the Dof domain region. The tree was analyzed as previously described but using 9.2 million generation run. Phylogenetic trees were visualized and edited using FigTree v.1.4 (http://tree.bio.ed.ac.uk/software/figtree/).

\section{Analysis of whole VviDofL transcriptome}

In order to fully characterize the expression of VviDofL genes during grapevine development, 54 tissues and organs of Vitis vinifera cv Corvina (clone 48, rootstock 41B) were analyzed using a comprehensive genome microarray. Plant materials used for the construction of the database encompassed all major grapevine organs in different developmental stages, and included data from berries during post-harvest withering, green and woody tissues, as well as specialized tissues such as pollen. Genome-wide transcriptome analysis was performed by microarray as described. ${ }^{49}$ The expression data was analyzed by hierarchical clustering on the whole 54-sample data set. Pearson's correlation distance was used as the metric, and T-MeV v4.81 (Boston, MA, USA) software was used to create the transcriptional profiles dendrograms for both genes and samples. ${ }^{50}$ Expression data are shown as normalized based on the mean center genes/rows adjustment.

Real-time PCR

Grapevine (Vitis vinifera L. cv Pinot Noir grafted on Paulsen 1103) samples from at least seven independent plants located in experimental plots at Embrapa Uva e Vinho (Bento Gonçalves/RS, Brazil) were sampled during the $2008 / 2009$ growing season. Three biological replicates consisted of tissues from three independent plants kept separately throughout the analysis. The developmental stages were defined as described by Coombe ${ }^{51}$ (modified E-L system) except when not indicated: roots of in vitro cultivated plants, $5 \mathrm{~cm}$ diameter leaves, tips of $10 \mathrm{~cm}$ stems (E-L 12), inflorescences and tendrils from pre-anthesis ( $E-L$ 17), summer buds (E-L 31), 7 mm-large berries ( $E-L$ 31), pre-véraison and véraison berries (E-L 34-35). Pre-véraison and véraison berries were sampled from the same bunch by separating half-colored berries from green colored berries. All samples were frozen in liquid nitrogen in the field and stored at $-80^{\circ} \mathrm{C}$ until RNA extraction.

Primer pairs were designed using the Primer3 program (http:// frodo.wi.mit.edu/primer3, Supplementary Table S2). Total RNA was extracted from frozen tissues using the Purelink RNA Reagent (Invitrogen, Carlsbad, CA, USA) and protocols recommended by the manufacturer. All RNA preparations were treated with DNAse (Fermentas, Waltham, MA, USA) in order to eliminate residual DNA contamination. Quantity and quality of total RNAs were evaluated with the Qubit Quantitation Platform (Invitrogen) and standard $1.5 \%$ agarose gel electrophoresis in $1 \times$ MOPS buffer. Complementary DNA was prepared using M-MLV Reverse Transcriptase (Promega, Madison, WI, USA). Transcript levels were determined by real-time PCR using a StepOne Plus PCR system (Applied Biosystems, Foster City, CA, USA) and SYBR Green (Applied Biosystems). The cycling protocol consisted of one step at $95^{\circ} \mathrm{C}$ for $5 \mathrm{~min}$, followed by 40 cycles at $95^{\circ} \mathrm{C}$ for $15 \mathrm{~s}$ and $60^{\circ} \mathrm{C}$ for $35 \mathrm{~s}$, and finished by a dissociation curve between 60 and $95^{\circ} \mathrm{C}$. The specificity of PCR amplifications was assessed by the presence of a single peak in the melting curves. Biological samples $(n=3)$ were analyzed in three technical replicates. Glyceraldehyde 3-phosphate dehydrogenase and tubulin were used as reference genes $^{52}$ using the $2-\Delta \Delta \mathrm{Ct}$ method. ${ }^{53}$

\section{RESULTS}

Identification and annotation of grapevine DofL genes

In order to identify putative Dof proteins in grapevine, a VviDofL sequence previously identified by the group ${ }^{23}$ was used as query in BLAST searches, resulting in the identification of 25 genes possibly coding for Dof proteins. This result was further confirmed in a HMM search using the Dof-seed sequence available at the Pfam database (PF02701) against the grapevine genome database. Putative Dof genes were named according to Grimplet et al. ${ }^{54}$ using phylogenetic trees provided by Gramene ${ }^{55}$ (Figure 1). Grapevine Dof domains were aligned and all highly conserved residues described to the DNA-binding Dof domain were mapped in the 25 sequences (Supplementary Figure S1). All predicted gene models identified along with their chromosomal location and deduced protein length are presented in Figure 1. The putative functions of the grapevine Dof proteins were further investigated by predicting their subcellular localization using the Plant-mPLoc database. $^{45}$ Only three sequences (VviDofL23, VviDofL24 and VviDofL25) not had their predicted subcellular location to the nucleus (Supplementary Table S3). Finally, intron and exon structures, as well as Dof domains, were determined and are displayed in Supplementary Figure S2 using FancyGene v1.4. ${ }^{56}$

The 25 putative Dof genes were found to be distributed in 14 out of the 19 grapevine chromosomes, as well as in the unplaced contigs chromosome (ChrUn), which contains sequences whose 


\begin{tabular}{|c|c|c|c|c|}
\hline Gene name & Genome locus & Chromosomal location & Size (aa) & Motif distribution \\
\hline VviDofL1.4 & VIT_00s0652g00010 & chrUn:33581346..33582988 & 295 & $\square \square$ Dof $\square$ \\
\hline VviDofL1.6 & VIT_03s0063g01350 & chr3:4802389..4802910 & 173 & \\
\hline VviDofL2.1 & VIT_06s0004g03420 & chr6:4265287..4267184 & 296 & \\
\hline VviDofL3.5 & VIT_08s0105g00170 & chr8:7109736..7110548 & 270 & Dof \\
\hline VviDofL5.6 & VIT_17s0000g08290 & chr17:9361008..9362865 & 308 & \\
\hline VviDofL6 & VIT_17s0000g06310 & $\operatorname{chr17:6845904..6848869}$ & 473 & Dof \\
\hline VviDofL7 & VIT_01s0026g02580 & chrl:12212183..12215486 & 477 & Dof \\
\hline VviDofL8 & VIT_14s0108g00980 & chr14:29617368..29620681 & 511 & Dof \\
\hline VviDofL9 & VIT_08s0056g01230 & chr8:1937113..1940026 & 494 & $\square$ \\
\hline VviDofL10 & VIT_18s0001g15730 & chr18:9622093..9623242 & 281 & \\
\hline VviDofL11 & VIT_02s0025g02250 & $\operatorname{chr} 2: 2012768 . .2014765$ & 288 & \\
\hline VviDofL12 & VIT_16s0098g01420 & $\operatorname{chr} 16: 21635846 . .21637912$ & 287 & \\
\hline VviDofL13 & VIT_15s0046g00150 & chr15:17188767..17190484 & 316 & \\
\hline VviDofL14 & VIT_08s0007g00180 & chr8:14550709..14554703 & 345 & \\
\hline VviDofL15 & VIT_13s0019g01410 & chr13:3000612..3002046 & 337 & Dof \\
\hline VviDofL16 & VIT_06s0004g04520 & chr6:5492077..5493425 & 320 & Dof \\
\hline VviDofL17 & VIT_10s0003g00030 & $\operatorname{chr} 10: 1318306 . .1319562$ & 299 & \\
\hline VviDofL18 & VIT_17s0000g04850 & chr17:5240463..5241938 & 318 & \\
\hline VviDofL19 & VIT_00s0218g00040 & chrUn:13957686..13958702 & 338 & Dof \\
\hline VviDofL20 & VIT_00s0253g00060 & chrUn:17750019..17752319 & 352 & $\square$ \\
\hline VviDofL21 & VIT_10s0003g00040 & $\operatorname{chr} 10: 1335706 . .1337565$ & 308 & Dof \\
\hline VviDofL22 & VIT_09s0002g02490 & chr9:2271830..2273304 & 216 & Dof \\
\hline VviDofL23 & VIT_07s0255g00020 & chr7:14346778..14347676 & 220 & Dof \\
\hline VviDofL24 & VIT_18s0001g11310 & chr18:13865318..13866466 & 226 & Dof \\
\hline VviDofL25 & VIT_10s0003g01260 & chr10:2577215..2577834 & 159 & $\square$ Dof \\
\hline
\end{tabular}

Figure 1. Identification of grapevine DofL genes. Genome locus is provided by the 'Grape Genome' (http://genomes.cribi.unipd.it/). Chromosomal localization and deduced peptide lengths are shown. Schematic view of the conserved motifs between VviDofL-deduced protein sequences was performed by the MEME suite. ${ }^{43}$ Colored boxes represent conserved motifs (Supplementary Figure S3). The height of the motif box is proportional to - $\log \left(P\right.$ value), with the maximum height denoting a $P$ value of $e^{-10}$. Gray lines represent non-conserved sequences. VviDofL 18 was previously named VvDof1 by Costenaro-da-Silva et al. ${ }^{23}$

physical position on specific chromosomes have not yet been defined. As shown in Figure 2, chromosomes 8, 10 and 17 have three Dofl genes each, chromosomes 6 and 18 both have two DofL genes and chromosomes 1, 2, 3, 7, 9, 13, 14, 15 and 16 have one DofL gene each. ChrUn also contains three DofL genes. Genome annotated scaffolds and molecular markers mapped near to DofL genes in each grapevine chromosome are also represented in Figure 2.

Phylogenetic studies of predicted DofL proteins

The analysis of the deduced grapevine DofL protein sequences using the MEME software revealed the existence of several different motifs apart from the conserved Dof domain. Eleven motifs were identified with an e-value support lower than $1^{-10}$ and their annotation was performed using Pfam and SMART databases. However, only the Dof domain could be annotated (cyan motif in Figure 1 and in Supplementary Figure S3). The overall motif distribution of grapevine DofL sequences is shown in Figure 1. The Dof motif was the only one shared between all sequences. The other ten motifs were distributed unequally between DofL proteins (Figure 1). The consensus sequences of all motifs are shown in Supplementary Figure S3.

A phylogenetic study was carried out to evaluate evolutionary relationships within DofL genes. To this purpose, 167 sequences from 19 different plant species were used, including all identified sequences from Arabidopsis, grapevine, poplar and tomato, in addition to Dof proteins already described in barley, C. reinhardtii, J. curcas, maize, maritime pine, moss, pea, potato, pumpkin, rice, sorghum, soybean, sweet potato, tobacco and wheat (Supplementary Table S1). On the basis of previous phylogenetic analysis, ${ }^{18,22,26}$ the Dof sequence from C. reinhardtii was considered as a common Dof ancestor and was employed to root the tree. The phylogenetic tree generated by using full alignments produced high nodal support values (Figure 3 ). The tree topology 

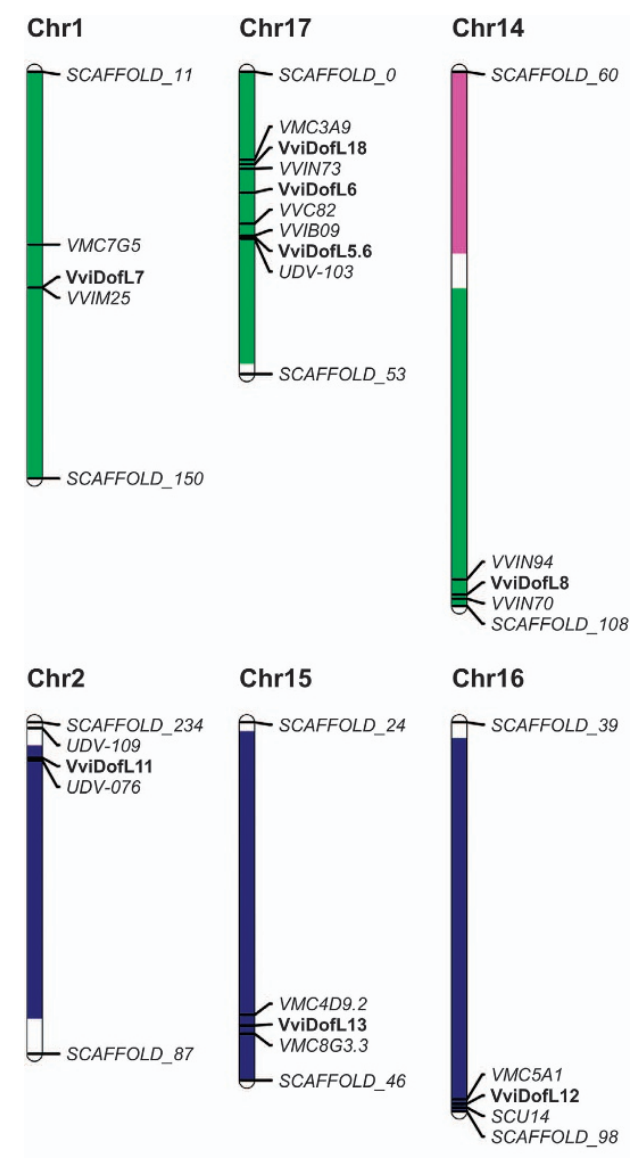

Chr7
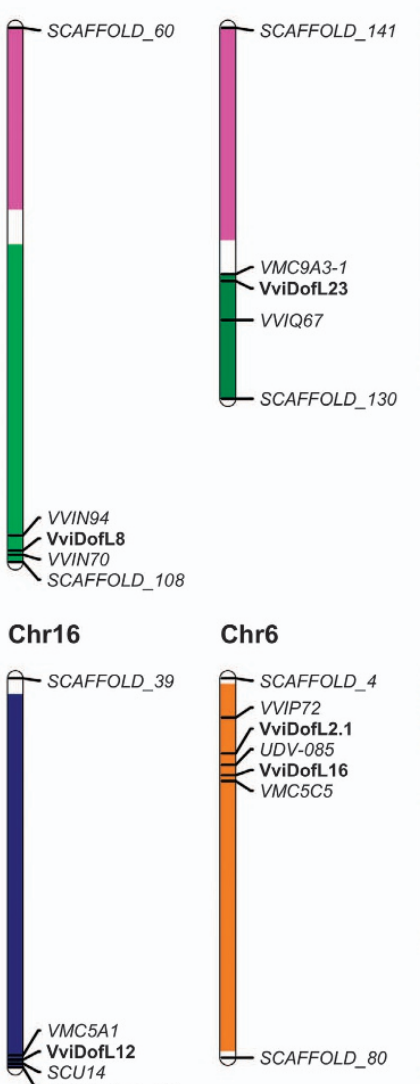

Chr3
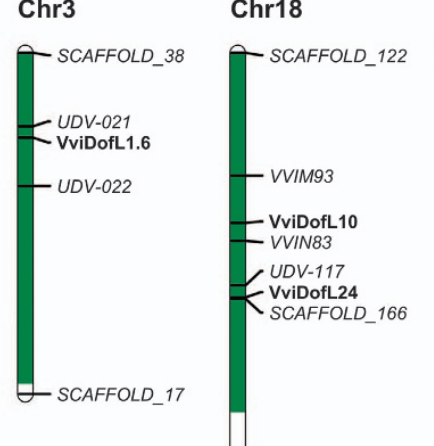

Chr9
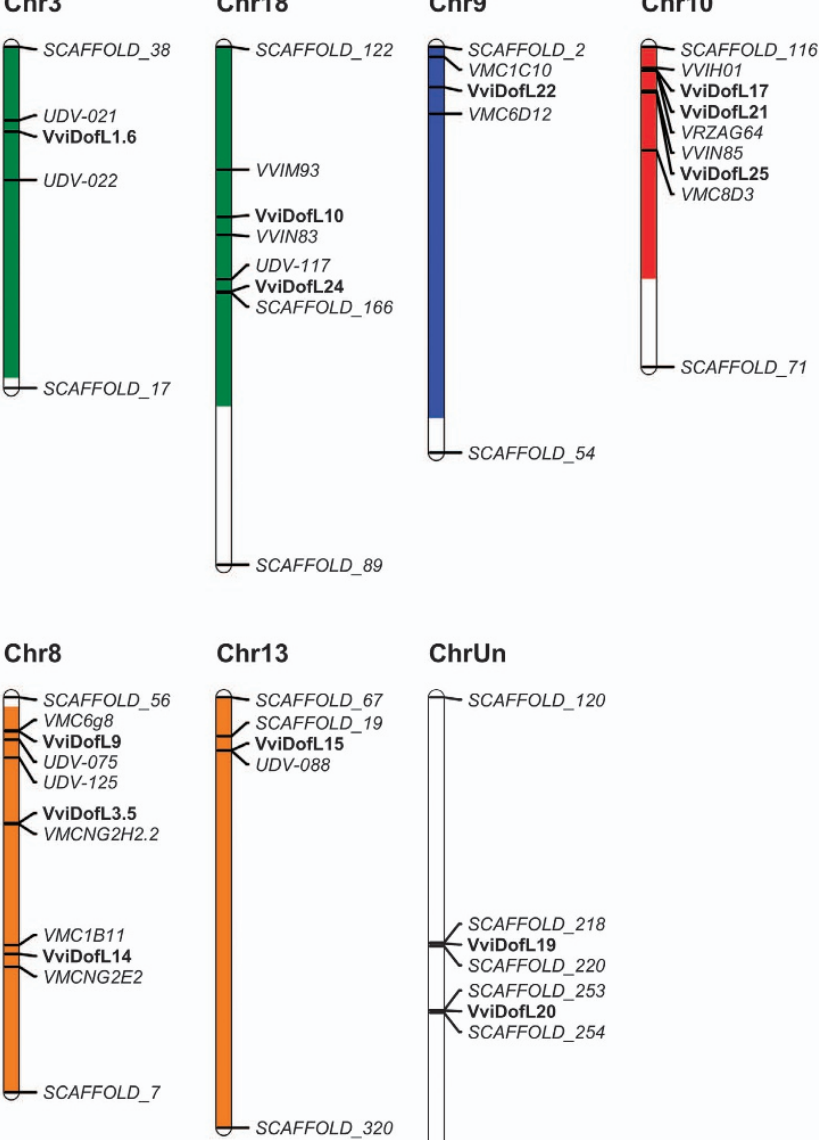

ChrUn

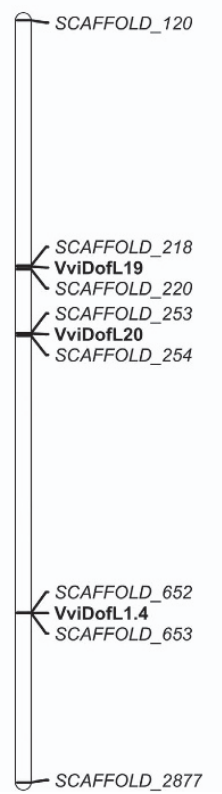

Figure 2. Chromosomal location of 25 VviDofL genes. Paralogous regions in the putative ancestral constituents of the grapevine genome are depicted in the color scheme following Jaillon et al. ${ }^{40}$ Molecular markers and scaffolds that help positioning genes are in italics.

obtained by the Bayesian Inference led to the identification of six major clusters of orthologous groups (MCOGs), numbered from I to VI. Interestingly, all MCOGs identified displayed at least one member from grapevine, poplar and tomato. Surprisingly, when curing the alignment, which converged to the Dof domain spanning region as the sole input sequence for the phylogenetic analysis, no trees with acceptable support values were produced (Supplementary Figure S4).

From the phylogenetic tree (Figure 3), 40 putative paralogs and 14 putative orthologs were identified among all 167 sequences analyzed. The majority of the paralogous pairs belonged to poplar (19) followed by Arabidopsis (10), tomato (9), barley and sorghum (one pair each). No paralogs were identified among grapevine sequences. Interestingly, eleven grapevine sequences formed clusters with paralogs from poplar (10) or tomato (1), namely VviDofL1.4, 5.6, 7, 9, 10, 11, 12, 16, 17, 20 and 23 (clades colored in black in Figure 3). Five putative orthologs were identified among grapevine: VviDofL1.6/PpiDof5, VviDofL22/PtDof19, VviDofL21/ PtDof20, VviDofL18/SIDof21 and VviDofL19/SIDof31 (these clades are collapsed in Figure 3). Finally, VviDofL2.1 formed a cluster with the orthologous pair GmDof11/PsDof1.

\section{Expression analysis of DofL genes}

The expression profiles of putative grapevine $V v i D o f L$ genes were retrieved from a global transcriptomic atlas comprising 54 tissues, organs or developmental stages. ${ }^{49}$ Overall, higher transcript accumulation was found in rachis, tendrils, buds and whole inflorescences, while low levels were identified in petals, stamens, pollen and berry skins. Hierarchical clustering analysis was carried out in order to find groups of VviDofL genes with similar transcript level profiles across samples. Groups were mostly composed of samples originated from the same tissue/organ (Figure 4). However, VviDofL expression patterns in buds, stems and berries were not totally clustered. A clear distinction could be observed between green/vegetative and mature/woody tissues and organs. In addition, senescing leaves presented marked differences in the patterns of $V$ viDofL gene expression relatively to other leaf samples.

Seven major patterns of VviDofL mRNA accumulation were identified through samples (A-G in Figure 4). Genes from group A presented higher transcript levels in several berry stages (mainly ripening to mid-ripening). In addition, VviDofL25 showed high expression in pollen, root, seedling and senescing leaves. Genes 


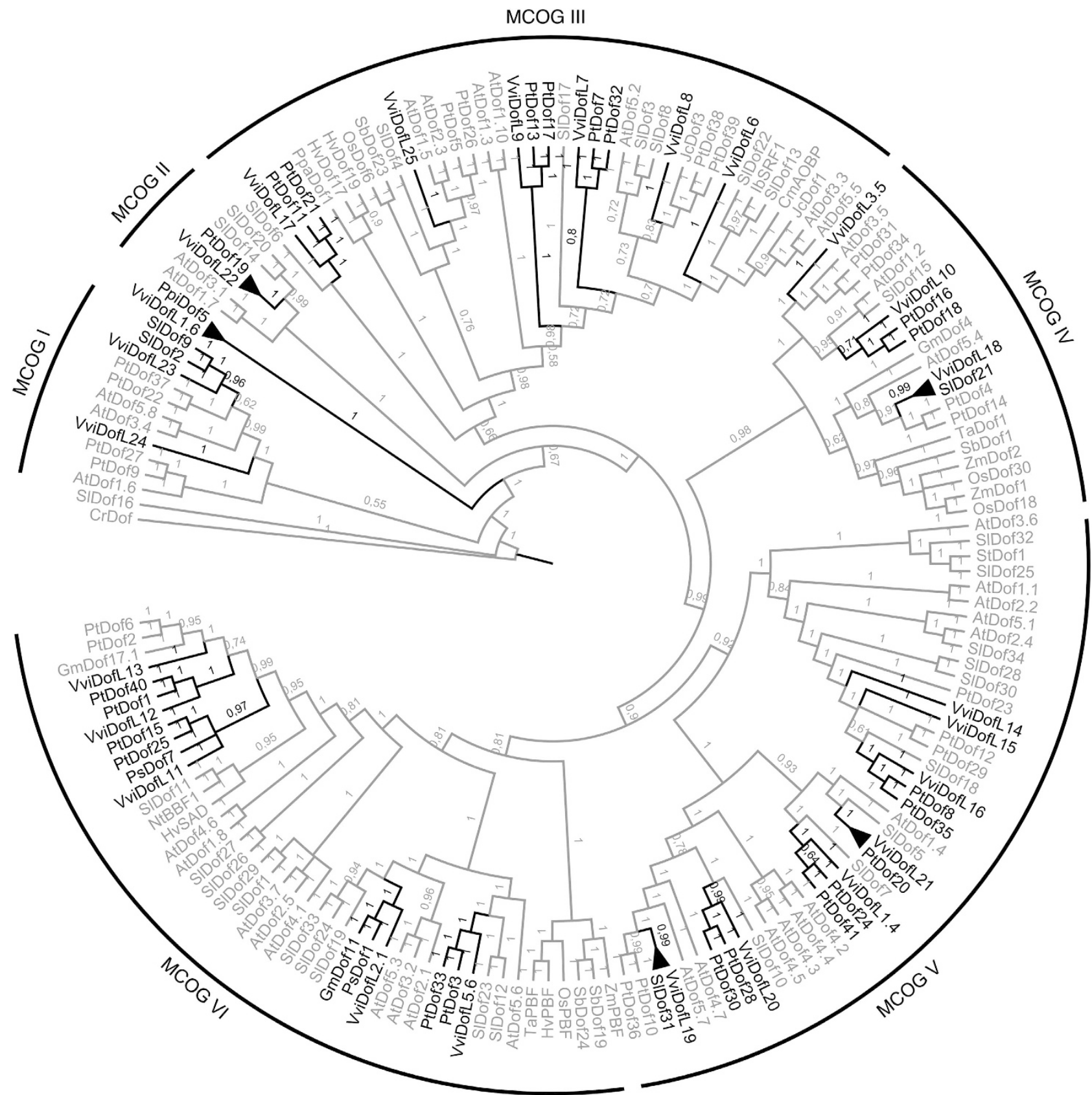

Figure 3. Phylogenetic tree of the full-length alignment of 167 Dof protein sequences. The resulting major clusters of orthologous genes (MCOGs) were numbered from I to VI. The tree was inferred using MrBayes v.3.2.6. ${ }^{47}$ Branch support is given by posteriori probability values shown next to the corresponding nodes (when $>0.50$ ). All accession codes used in the phylogenetic analysis are depicted in Supplementary Table S1. Clades comprising grapevine orthologs are collapsed; clades of grapevine proteins clusterized with paralogs or orthologs of other species are colored in black. At, Arabidopsis thaliana; Cm, Cucurbita maxima; Cr, C. reinhardtii; Gm, Glycine max; Hv, Hordeum vulgare; Ib, Ipomoea batatas; Jc, J. curcas; Nt, Nicotiana tabacum; Os, Oriza sativa; Ppa, P. patens; Ppi, Pinus pinaster; Ps, Pisum sativum; Pt, Populus trichocarpa; Sb, Sorghum bicolor; SI, Solanum lycopersicum; St, Solanum tuberosum; Ta, Triticum aestivum; Vv, V. vitifera; Zm, Zea mays.

from group B were mainly induced in inflorescences, buds and seeds at the stages of post-fruit set to ripening. Interestingly, genes from group $C$ showed low transcript amounts in all berry stages, except at the post-fruit set stage. Moreover, these genes were induced in tendril, rachis and bud stages. Transcripts from this group were presented in low levels in all four seed stages analyzed, with the exception of VviDofL20, VviDofL22 and VviDofL24 that were induced in seed at the fruit set and postfruit set stages. Considering the three genes that integrate group D, only VviDofL5.6 and VviDofL 16 were induced in rachis and berries from véraison to ripening stages, with VviDofL5.6 showing the highest expression levels. Genes from group $E$ and $F$ showed minor expression variation in the samples analyzed. However, VviDof 10 was highly induced in petals, stamens and flowers. Finally, genes from group $\mathrm{G}$ were mainly induced in berry stages (véraison to post-harvest withering III), with VviDofL13 and VviDofL 14 showing the highest expression levels. In summary, a broad expression pattern of VviDofL genes was found across the 54 grapevine developmental stages analyzed.

In order to complement the whole-transcriptome data, steadystate mRNA levels of all VviDofL genes were investigated by realtime PCR in nine grapevine organs and tissues of the Pinot Noir 


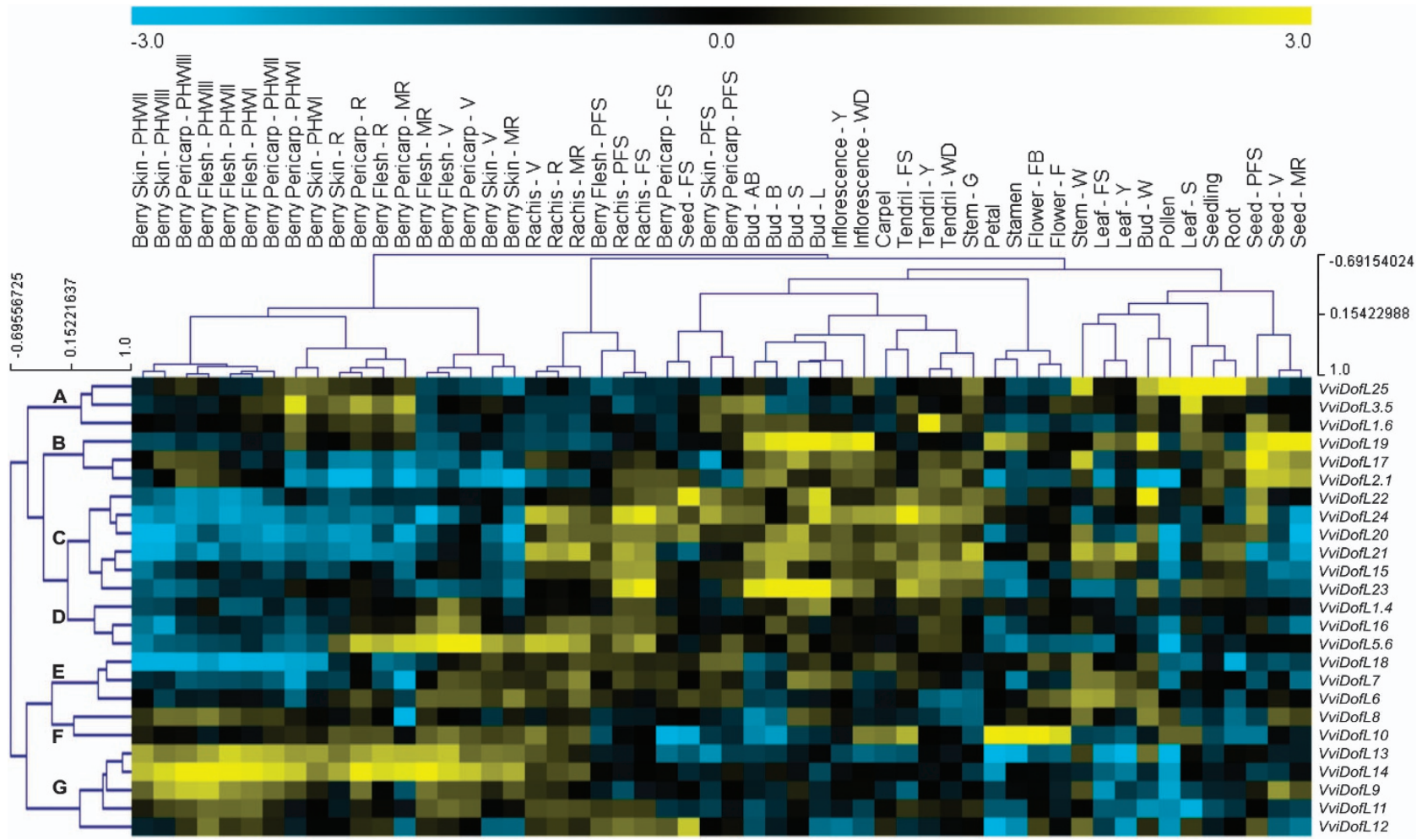

Figure 4. Transcript levels of VviDofL genes across 54 several grapevine cv Corvina tissues and organs. The color scheme used to represent expression level is yellow/blue: black boxes indicate low variation in gene expression, blue boxes indicate a fold decrease, and yellow boxes indicate a fold increase in relation to the mean value. Samples and genes were hierarchically clustered based on the average Pearson's distance, resulting in seven major patterns of VviDofL mRNA levels (A-G). Abbreviations in the sample headline: for buds: $A B$, after-burst; $B$, bud burst; L, latent bud; S, bud swell; W, winter bud; for inflorescences: WD, well developed; $Y$, young; for flowers: $F$, flowering; FB, flowering begins; for tendrils: FS, mature; WD, well developed; Y, young; for leaves: FS, mature; S, senescing; Y, young; for berry pericarp, berry skin, berry flesh, seed and rachis: FS, fruit set; MR, mid-ripening; PFS, post-fruit set; PHWI, post-harvest withering (first month); PHWII, post-harvest withering (second month); PHWII, post-harvest withering (third month); R, ripening; V, véraison; for stems: G, green; W, woody. Data obtained as described in Fasoli et al. ${ }^{49}$

grapevine variety. The overall grapevine Dof gene expression was quite diverse in these samples (Figure 5). Eleven VviDofL genes exhibited their highest expressions levels in inflorescences ( $V v i$ DofL3.5, VviDofL8, VviDofL10, VviDofL14, VviDofL16, VviDofL17, VviDofL18, VviDofL19, VviDofL20, VviDofL21, and VviDofL24), three in stems (VviDofL6, VviDofL7 and VviDofL23), two in stems and inflorescences (VviDofL1.4 and VviDofL2.1), one gene in summer buds and inflorescences (VviDof $L 22)$, one in inflorescences, prevéraison and véraison berries (VviDofL5.6), one in roots (VviDofL25), one in pre-véraison berries (VviDofL1.6), and one in véraison berries (VviDofL13). The genes with the highest expression specificity in the grapevine organs tested were VviDofL6, VviDofL8, VviDofL10, VviDofL19 and VviDofL25. None of the VviDofL genes seemed to be specific to tendrils or leaves, or to have any pronounced level of mRNA accumulation in these organs. After several trials of reamplification, no measurable amplicons for VviDofL9, VviDofL11, VviDofL12 and VviDofL15 was obtained (Figure 5).

\section{DISCUSSION}

Velasco et al. $^{39}$ identified 62 families of transcription factors in the consensus genome sequence of the heterozygous grapevine 'Pinot Noir' and, among them, Dof transcription factors. Corroborating those findings, we were able to identify, phylogenetically characterize, and describe the patterns of steady-state mRNA levels of 25 grapevine Dof genes. Among the seeded plant genomes annotated for Dof genes so far, grapevine, sugarcane and pine showed the smallest numbers. ${ }^{21,22}$ Although 25 putative Dof genes were found in grapevine and sugarcane, ${ }^{33}$ only 10 were identified in pine. ${ }^{27,30}$ Other species showed more members for this gene family, ranging from 26 in barley to 78 in soybean. ${ }^{21,22,26,32}$

In the present work, the four characteristic cysteine residues for zinc docking, as well as other well conserved sequences, were identified in all 25 grapevine DofL proteins, suggesting that their Dof domains are functional (Supplementary Figure S1). Additionally, the predicted physical localization of DofL genes in grapevine chromosomes was presented (Figure 2). In addition to the Dof domain, a plethora of other motifs is shared among DofL proteins (Figure 1). This finding was already observed in several other reports. ${ }^{22,24-26,29-31}$ The presence, absence or position of these motifs may affect the functional role of each Dof transcription factor. However, no similarity with known domains was found when comparing these sequences with the Pfam or SMART databases. Surprisingly, the comparison of the 10 grapevine nonDof motifs identified (Supplementary Figure S3) with non-Dof motifs of other studies $22,24-26,29-31$ revealed similarities between the studies. This finding reinforces the need to conduct further investigations to unveil the functional roles of these still uncharacterized domains.

Comparative genomic studies are able to track characteristic features in multiple genomes. The identification of orthologous and paralogous sequences, along with functional information, can be useful tools to predict gene function. ${ }^{57,58}$ Moreover, gene duplication events are a common feature during genome and gene family evolution, and this seems to be the case in the Dof expansion. Moreno-Risueno et al. ${ }^{26}$ suggested that duplication events from an ancestral Dof gene would have triggered the expansion of this family, resulting in neo-, sub- and 
VviDofL1.4

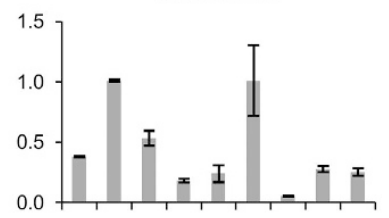

VviDofL5.6

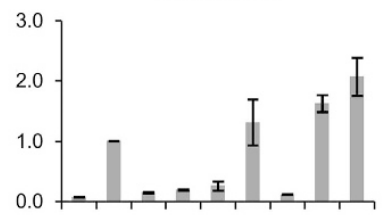

VviDofL10

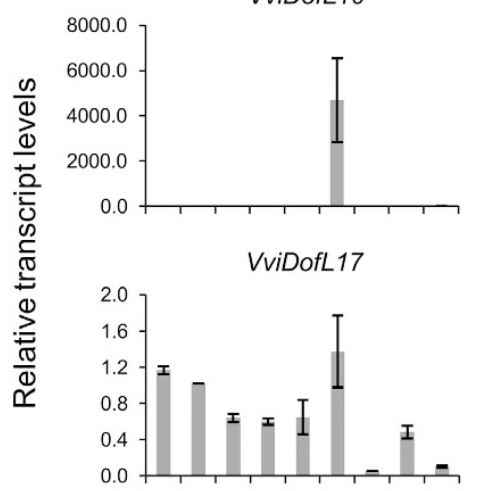

VviDofL21
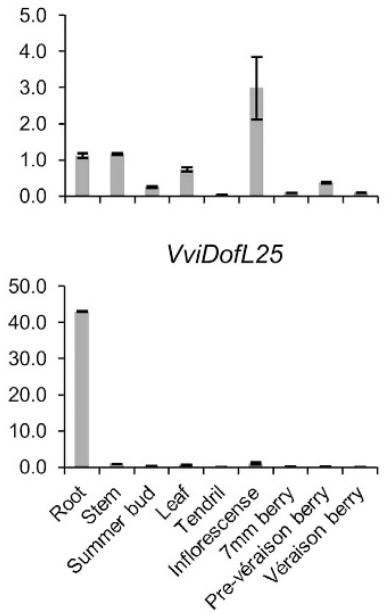

VviDofL1.6

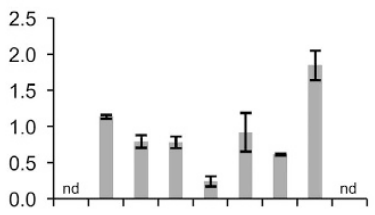

VviDofL6

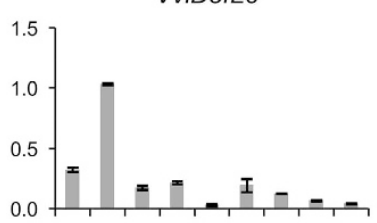

VviDofL13

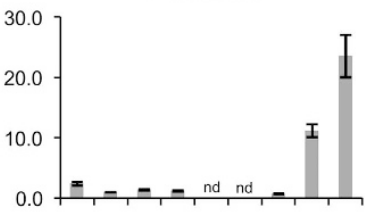

VviDofL18

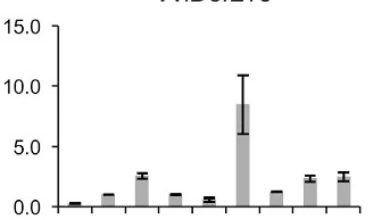

VviDofL22

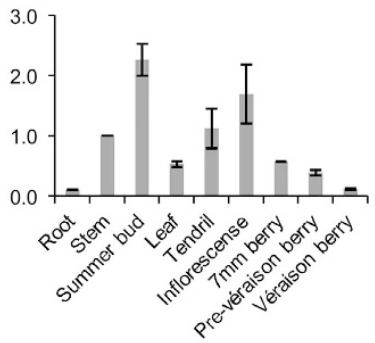

VviDofL2.1

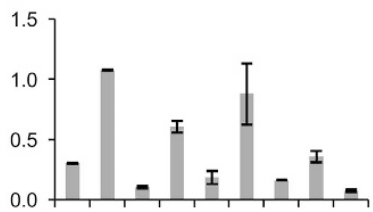

VviDofL7

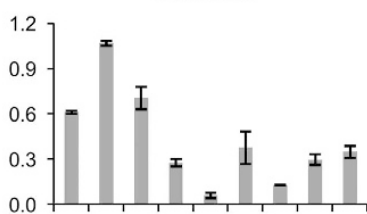

VviDofL14

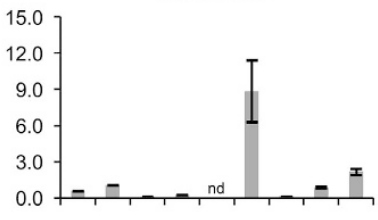

VviDofL19

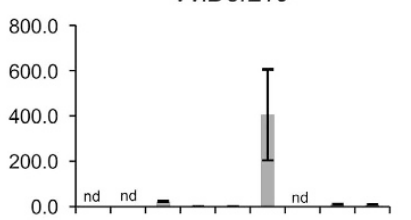

VviDofL23

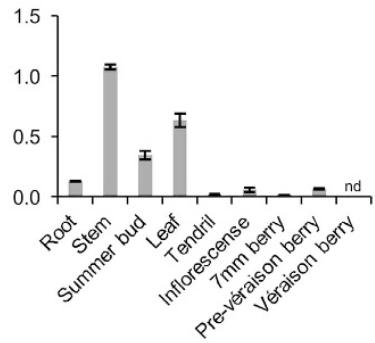

VviDofL3.5

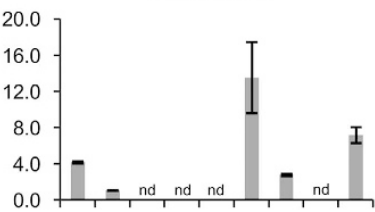

VviDofL8

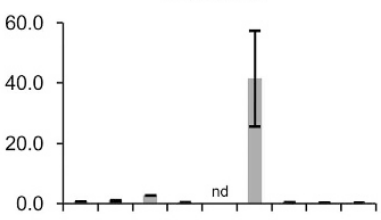

VviDofL16

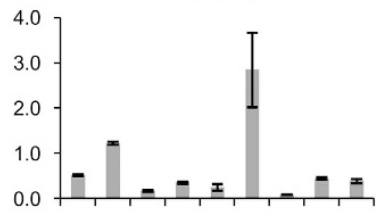

VviDofL20

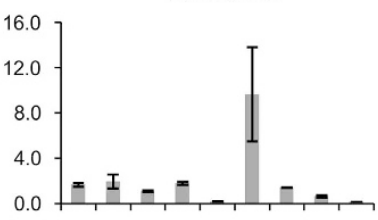

VviDofL24

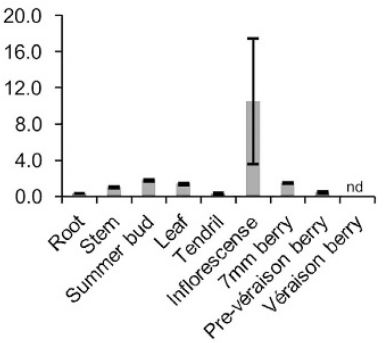

Figure 5. Expression profiles of VviDofL genes in nine grapevine cv Pinot Noir vegetative and reproductive organs by real-time PCR. Relative transcript levels in stems were set to 1, except VviDofL 19 whose leaf samples were set to 1. ND, non-detected. Standard error bars are shown.

pseudogenization processes that likely lead to their high range of functions. Indeed, Dof genes are exclusive of the green lineage, increasing from a single-copy gene in C. reinhardtii to a multicopy family with numerous functions in seeded plants. ${ }^{18}$ In this scenario, the identification of paralogous and orthologous genes along with functional information about Dof family members can be used to infer gene function, although further investigations are required to confirm these predictions.

Dof sequences from grapevine, Arabidopsis, poplar and tomato, in addition to Dof proteins already described in other species, were compared using a phylogenetic approach with the aim of improving the knowledge about the function of the VviDofL genes (Figure 3). The results fit well with previous reports, given that our analysis rendered the same 22 Dof paralogous and orthologous pairs identified by Yang et al., ${ }^{25} 11$ identified by Yanagisawa ${ }^{1}$ and 7 identified by Cai et al.. ${ }^{31}$ Its worth to mention that several other pairs identified by these groups formed new paralogs or orthologs in our set of sequences. This finding can be explained by the large set of sequences used in our analysis. As one could expect in a comparative genome analysis, the number of orthologous sequences found between grapevine and poplar (12 pairs; Figure 3) was higher than the one found between poplar and rice (four pairs in Yang et al. ${ }^{25}$ ). Additionally, the strategy of comparing Dof genes from species with established functions yielded four pairs of orthologs besides the ones with poplar (Figure 3). These Dof genes were already characterized in maritime pine and tomato and may be an important resource to better understand the VviDofL functions in grapevine. Interestingly, 
grapevine sequences did not form paralogs suggesting that neofunctionalization events could be held accountable in the expansion of this gene family in grapevine. As several authors have previously observed, ${ }^{24-26}$ the Dof domain is extremely well conserved among proteins and, for phylogenetic comparisons, the only strategy that generate consistent trees was based on sequences outside the Dof domain. In accordance, curing of the protein alignment was not effective in producing betterstructured trees given that the Bayesian algorithm was not able to branch the tree (Supplementary Figure S4). Lijavetzky et al. ${ }^{24}$ recognized that several clusters remained with poor supporting values in their phylogenetic analysis as the consequence of using only the conserved Dof domain (50 amino acids in length) in alignments.

Genome-wide studies describing the transcript accumulation patterns of Dof genes are found in the literature, reporting differential expression of Dof genes among tissues, organs or in response to abiotic stresses, ${ }^{25,26,28-31,34-37,59}$ but little information is available for grapevine Dof genes. ${ }^{23}$ In this work, by exploring genome-wide analysis by microarray in combination with real-time PCR, specific VviDofL transcriptional patterns were found. Transcriptome data obtained from various grapevine samples showed different VviDofL expression signatures according to the tissue/ organ tested (Figure 4). Interestingly, some tissues/organs and mature/woody samples tended to display transcript accumulation profiles considerably distant from their vegetative/green counterparts. This trend was also observed for the whole set of organspecific grapevine genes when screened for mRNA levels in the same samples. ${ }^{49}$ Furthermore, several expression profiles were obtained by real-time PCR (Figure 5) and support the microarray data. This finding is very interesting given that the plant material used in the two approaches is highly divergent, suggesting their involvement in similar processes regardless the cultivar. Finally, these results indicate that functional roles are being played by VviDofL genes during specific developmental stages, especially in reproductive tissues.

A recent review on the subject highlighted the importance of Dof genes during three main processes: metabolism regulation, seed development and tissue differentiation. ${ }^{22}$ The whole transcriptome data strongly suggest a clear relationship between VviDofl genes and the development of berries, seeds and flowers. Although most VviDofL genes presented low levels of transcripts during berry development (Figure 4), genes from group G, mainly VviDofL13 and VviDofL14, were highly induced in berries from véraison to post-harvest withering III stages. In addition, VviDofL3.5 (group A) displayed higher expression levels in berries at midripening to ripening stages, and VviDofL5.6 (group D) showed high transcript levels in berries from véraison to ripening stages. Similar results were obtained by real-time PCR for VviDofL5.6 and VviDofL13 (Figure 5). Costenaro-da-Silva et al. ${ }^{23}$ reported a DofL gene (VviDofL18 in this work) being induced during berry developmental stages 2-6 weeks after fruit set; however, this relationship still lacks an in-depth characterization. Berry development in grapevine is a finely tuned complex process, and the results described in this work suggest that VviDofL3.5, 5.6, 13 and 14 are important players in the regulation of berry development in grapevine. Complementarily, there is a widely accepted positive correlation between berry weight and seed content in grapevine segregating populations, possibly due to the action of growth regulators produced by seeds. ${ }^{60}$ In this respect, seeds can influence berry development, also having important roles in enological traits, given that seeds are one of the major providers of tannins during berry growth. ${ }^{61}$ Indeed, several VviDofL genes were highly induced in seed samples and they may be playing roles during seed development. This seems to be the case of genes from group B and genes VviDofL20, VviDofL22 and VviDofL24 from group C (Figure 4). These genes presented a remarkable accumulation of transcripts in all seed stages analyzed (fruit set to mid-ripening) and might be acting together in seed development. Interestingly, VviDofL2.1 from group B, which was almost exclusively expressed in seeds from post-fruit set to midripening stages (Figure 4), exhibited orthology with GmDof11 and PsDof1 (Figure 3). GmDof11, along with GmDof4, is involved in the regulation of genes associated with the synthesis of fatty acids, controlling the accumulation of lipids in soybean seeds. ${ }^{59}$ It is tempting to speculate that GmDof11 and VviDofL2.1 share conserved functions, although functional studies are needed to confirm or refute these statements. Furthermore, several functional studies reported the involvement of Dof proteins in the regulation of seed content, germination and/or development. Dof proteins from barley (HvSAD and HvPBF), maize (ZmPBF) and rice (OsPBF) were shown to activate several genes during seed development that are responsible for $15 \%$ of the total endosperm protein content. ${ }^{10-14}$ These Dof transcription factors also participate on the mobilization of storage compounds in order to provide nutrients for seed germination and seedling growth. ${ }^{8}$ In addition, DAG1 (Dof Affecting Germination 1) and DAG2 act on a maternal switch that is responsible for controlling seed germination in Arabidopsis. ${ }^{3-6}$ In this context, the transcriptional data gathered in this work suggest that several VviDofL genes are playing important roles during the course of berry and seed development.

Eleven VviDofL genes were mainly expressed in inflorescences when screened for mRNA levels by real-time PCR (Figure 5), and VviDofL10, VviDofL19 and VviDofL24 also presented floweringspecific transcription profiles in the whole transcriptome data (Figure 4). The identification of similar expression profiles between microarray and real-time PCR approaches, comparing samples from different cultivars, orchard management practices and climate growth conditions, strongly suggest that these genes have important regulatory roles during flowering that may be evolutionally conserved between grapevine varieties. In the literature, few reports about Dof functions during flowering are found, ${ }^{19,20,62}$ although some Dofs were described by their involvement in daylength regulation of flowering through interactions with CONSTANS. ${ }^{15-18}$ However, inside a subclade of MCOG V, VviDofL19 is very close to AtDof4.7 (Figure 3), which is involved in the control of floral abscission as part of a transcription complex that directly regulates the expression of cell wall hydrolysis enzymes. ${ }^{19}$ Moreover, ZmDof1 from maize was described as a transcriptional repressor of pollen development. ${ }^{20}$ Finally, flower-specific expression of Dof genes was already identified for AtDof4.2, which negatively and positively regulates the transcription of flavonoid and hydroxycinnamic acid production, respectively. ${ }^{62}$ Taken together, our findings strongly suggest that several VviDofLs might be acting in specific functions during flower development. Although promising, the genes herein identified still need further investigations in order to unveil their functional roles.

In conclusion, we identified the probable full set of DofL genes in grapevine. The phylogenetic analysis resulted in the identification of seven MCOGs that contains members from 18 plant species. Our results confirm that recurrent duplications and diversification from an original Dof ancestor led to the formation of this complex family of transcription factors specific to Viridiplantae. In view of important genome duplication events leading to gene redundancy in the history of plant diversification (see Jaillon et al. ${ }^{40}$ for example), a combination of phylogenetically inferred relationships with functional data will be essential to effectively establish conserved and diverged roles of the DofL gene family in grapevine and in other plant species. Finally, our expression data revealed the involvement of $V$ viDof $L$ genes mainly in berry, seed and flower development in grapevine. In this work, we gathered consistent results that turn the VviDofL genes into good candidates to better understand the regulatory network associated with flowering and berry development. 


\section{CONFLICT OF INTEREST}

The authors declare no conflict of interest.

\section{ACKNOWLEDGEMENTS}

We thank Dr Roberto C Togawa for providing us with the access to the Bioinformatics Laboratory at Embrapa Recursos Genéticos e Biotecnologia. This work was supported by 'Coordenação de Aperfeiçoamento de Pessoal de Nível Superior' (CAPES, Brazil), 'Conselho Nacional de Desenvolvimento Científico e Tecnológico (CNPq, Brazil; Proc. 475440/2004-8; GP research fellowship 311361/2009-9), 'Fundação de Amparo à Pesquisa do Estado do Rio Grande do Sul' (FAPERGS, Brazil; Proc. 04/1184.6) and Embrapa (Brazil).

\section{REFERENCES}

1 Yanagisawa S. The Dof family of plant transcription factors. Trends Plant Sci 2002; 7: $555-560$.

2 Le Hir R, Bellini C. The plant-specific Dof transcription factors family: new players involved in vascular system development and functioning in Arabidopsis. Front Plant Sci 2013; 4: 164

3 Gabriele S, Rizza A, Martone J, Circelli P, Costantino P, Vittorioso P. The Dof protein DAG1 mediates PIL5 activity on seed germination by negatively regulating GA biosynthetic gene AtGA3ox1. Plant J 2010; 61: 312-323.

4 Gualberti G, Papi M, Bellucci L, Ricci I, Bouchez D, Camilleri C et al. Mutations in the Dof zinc finger genes DAG2 and DAG1 influence with opposite effects the germination of Arabidopsis seeds. Plant Cell 2002; 14: 1253-1263.

5 Papi M, Sabatini S, Bouchez D, Camilleri C, Costantino P, Vittorioso P. Identification and disruption of an Arabidopsis zinc finger gene controlling seed germination. Genes Dev 2000; 14: 28-33.

6 Santopolo S, Boccaccini A, Lorrai R, Ruta V, Capauto D, Minutello E et al. DOF AFFECTING GERMINATION 2 is a positive regulator of light-mediated seed germination and is repressed by DOF AFFECTING GERMINATION 1. BMC Plant Biol 2015; 15: 72.

7 Isabel-LaMoneda I, Diaz I, Martinez M, Mena M, Carbonero P. SAD: a new DOF protein from barley that activates transcription of a cathepsin B-like thiol protease gene in the aleurone of germinating seeds. Plant J 2003; 33: 329-340.

8 Martínez M, Rubio-Somoza I, Fuentes R, Lara P, Carbonero P, Díaz I. The barley cystatin gene (Icy) is regulated by DOF transcription factors in aleurone cells upon germination. J Exp Bot 2005; 56: 547-556.

9 Rueda-Romero P, Barrero-Sicilia C, Gómez-Cadenas A, Carbonero P, Oñate-Sánchez L. Arabidopsis thaliana DOF6 negatively affects germination in non-afterripened seeds and interacts with TCP14. J Exp Bot 2012; 63: 1937-1949.

10 Diaz I, Martinez M, Isabel-LaMoneda I, Rubio-Somoza I, Carbonero P. The DOF protein, SAD, interacts with GAMYB in plant nuclei and activates transcription of endosperm-specific genes during barley seed development. Plant $J$ 2005; 42: 652-662.

11 Dong G, Ni Z, Yao Y, Nie X, Sun Q. Wheat Dof transcription factor WPBF interacts with TaQM and activates transcription of an alpha-gliadin gene during wheat seed development. Plant Mol Biol 2007; 63: 73-84.

12 Hwang Y-S, Ciceri P, Parsons RL, Moose SP, Schmidt RJ, Huang N. The maize O2 and $\mathrm{PBF}$ proteins act additively to promote transcription from storage protein gene promoters in rice endosperm cells. Plant Cell Physiol 2004; 45: 1509-1518.

13 Marzábal P, Gas E, Fontanet P, Vicente-Carbajosa J, Torrent M, Ludevid MD. The maize Dof protein PBF activates transcription of gamma-zein during maize seed development. Plant Mol Biol 2008; 67: 441-454.

14 Yamamoto MP, Onodera Y, Touno SM, Takaiwa F. Synergism between RPBF Dof and RISBZ1 bZIP activators in the regulation of rice seed expression genes. Plant Physiol 2006; 141: 1694-1707.

15 Fornara F, Panigrahi KCS, Gissot L, Sauerbrunn N, Ruhl M, Jarillo JA et al. Arabidopsis DOF transcription factors act redundantly to reduce CONSTANS expression and are essential for a photoperiodic flowering response. Dev Cell 2009; 17: 75-86.

16 Imaizumi T, Schultz TF, Harmon FG, Ho LA, Kay SA. FKF1 F-box protein mediates cyclic degradation of a repressor of CONSTANS in Arabidopsis. Science 2005; 309: 293-297.

17 Li D, Yang C, Li X, Gan Q, Zhao X, Zhu L. Functional characterization of rice OsDof12. Planta 2009; 229: 1159-1169.

18 Lucas-Reina E, Romero-Campero FJ, Romero JM, Valverde F. An evolutionarily conserved DOF-CONSTANS module controls plant photoperiodic signaling. Plant Physiol 2015; 168: 561-574.

19 Wei P-C, Tan F, Gao X-Q, Zhang X-Q, Wang G-Q, Xu H et al. Overexpression of AtDOF4.7, an Arabidopsis DOF family transcription factor, induces floral organ abscission deficiency in Arabidopsis. Plant Physiol 2010; 153: 1031-1045.
20 Chen X, Wang D, Liu C, Wang M, Wang T, Zhao Q et al. Maize transcription factor Zmdof1 involves in the regulation of Zm401 gene. Plant Growth Regul 2012; 66: 271-284.

21 Gupta S, Malviya N, Kushwaha H, Nasim J, Bisht NC, Singh VK et al. Insights into structural and functional diversity of Dof (DNA binding with one finger) transcription factor. Planta 2015; 241: 549-562.

22 Noguero M, Atif RM, Ochatt S, Thompson RD. The role of the DNA-binding One Zinc Finger (DOF) transcription factor family in plants. Plant Sci 2013; 209: 32-45.

23 Costenaro-da-Silva D, Passaia G, Henriques JAP, Margis R, Pasquali G, Revers LF. Identification and expression analysis of genes associated with the early berry development in the seedless grapevine (Vitis vinifera L.) cultivar Sultanine. Plant Sci 2010; 179: 510-519.

24 Lijavetzky D, Carbonero P, Vicente-Carbajosa J. Genome-wide comparative phylogenetic analysis of the rice and Arabidopsis Dof gene families. BMC Evol Biol 2003; 3: 17.

25 Yang X, Tuskan GA, Cheng Z-M. Divergence of the Dof gene families in poplar, Arabidopsis, and rice suggests multiple modes of gene evolution after duplication. Plant Physiol 2006; 142: 820-830.

26 Moreno-Risueno MA, Martínez M, Vicente-Carbajosa J, Carbonero P. The family of DOF transcription factors: from green unicellular algae to vascular plants. $\mathrm{Mol}$ Genet Genomics 2007; 277: 379-390.

27 Shigyo M, Tabei N, Yoneyama T, Yanagisawa S. Evolutionary processes during the formation of the plant-specific Dof transcription factor family. Plant Cell Physiol 2007; 48: 179-185.

28 Shaw LM, McIntyre CL, Gresshoff PM, Xue G-P. Members of the Dof transcription factor family in Triticum aestivum are associated with light-mediated gene regulation. Funct Integr Genomics 2009; 9: 485-498.

29 Hernando-Amado S, González-Calle V, Carbonero P, Barrero-Sicilia C. The family of DOF transcription factors in Brachypodium distachyon: phylogenetic comparison with rice and barley DOFs and expression profiling. BMC Plant Biol 2012; 12: 202.

30 Rueda-López M, García-Gutiérrez A, Cánovas FM, Ávila C. The family of Dof transcription factors in pine. Trees 2013; 27: 1547-1557.

31 Cai X, Zhang Y, Zhang C, Zhang T, Hu T, Ye J et al. Genome-wide analysis of plantspecific Dof transcription factor family in tomato. J Integr Plant Biol 2013; 55: 552-566.

32 Guo Y, Qiu L-J. Genome-wide analysis of the Dof transcription factor gene family reveals soybean-specific duplicable and functional characteristics. PLoS One 2013; 8: e76809.

33 Gupta S, Kushwaha H, Singh VK, Bisht NC, Sarangi BK, Yadav D. Genome wide in silico characterization of Dof transcription factor gene family of sugarcane and its comparative phylogenetic analysis with Arabidopsis, rice and sorghum. Sugar Tech 2014; 16: 372-384.

34 Ma J, Li M-Y, Wang F, Tang J, Xiong A-S. Genome-wide analysis of Dof family transcription factors and their responses to abiotic stresses in Chinese cabbage. BMC Genomics 2015; 16: 33.

35 Chen Y, Cao J. Comparative analysis of Dof transcription factor family in maize. Plant Mol Biol Report 2015; 33: 1245-1258.

36 Venkatesh J, Park SW. Genome-wide analysis and expression profiling of DNAbinding with one zinc finger (Dof) transcription factor family in potato. Plant Physiol Biochem 2015; 94: 73-85.

37 Huang W, Huang Y, Li M, Wang F, Xu Z, Xiong A. Dof transcription factors in carrot: genome-wide analysis and their response to abiotic stress. Biotechnol Lett 2016; 38: $145-155$.

38 Altschul SF, Gish W, Miller W, Myers EW, Lipman DJ. Basic local alignment search tool. J Mol Biol 1990; 215: 403-410.

39 Velasco R, Zharkikh A, Troggio M, Cartwright DA, Cestaro A, Pruss D et al. A high quality draft consensus sequence of the genome of a heterozygous grapevine variety. PLoS One 2007; 2: e1326.

40 Jaillon O, Aury J-M, Noel B, Policriti A, Clepet C, Casagrande A et al. The grapevine genome sequence suggests ancestral hexaploidization in major angiosperm phyla. Nature 2007; 449: 463-467.

41 Punta M, Coggill PC, Eberhardt RY, Mistry J, Tate J, Boursnell C et al. The Pfam protein families database. Nucleic Acids Res 2012; 40: D290-D301.

42 Voorrips RE. MapChart: software for the graphical presentation of linkage maps and QTLs. J Hered 2002; 93: 77-78.

43 Bailey TL, Boden M, Buske F a, Frith M, Grant CE, Clementi L et al. MEME SUITE: tools for motif discovery and searching. Nucleic Acids Res 2009; 37: W202-W208.

44 Letunic I, Doerks T, Bork P. SMART 7: recent updates to the protein domain annotation resource. Nucleic Acids Res 2012; 40: D302-D305.

45 Chou KC, Shen HB. Plant-mPLoc: A top-down strategy to augment the power for predicting plant protein subcellular localization. PLoS One 2010; 5: e11335.

46 Thompson JD, Higgins DG, Gibson TJ. CLUSTAL W: improving the sensitivity of progressive multiple sequence alignment through sequence weighting, positionspecific gap penalties and weight matrix choice. Nucleic Acids Res 1994; 22: 4673-4680. 
47 Ronquist F, Teslenko M, van der Mark P, Ayres DL, Darling A, Hohna S et al. MrBayes 3.2: Efficient Bayesian phylogenetic inference and model choice across a large model space. Syst Biol 2012; 61: 539-542.

48 Castresana J. Selection of conserved blocks from multiple alignments for their use in phylogenetic analysis. Mol Biol Evol 2000; 17: 540-552.

49 Fasoli M, Dal Santo S, Zenoni S, Tornielli GB, Farina L, Zamboni A et al. The grapevine expression atlas reveals a deep transcriptome shift driving the entire plant into a maturation program. Plant Cell 2012; 24: 3489-3505.

50 Saeed Al, Bhagabati NK, Braisted JC, Liang W, Sharov V, Howe EA et al. TM4 microarray software suite. Methods Enzymol 2006; 411: 134-193.

51 Coombe BG. Growth stages of the grapevine: Adoption of a system for identifying grapevine growth stages. Aust J Grape Wine Res 1995; 1: 104-110.

52 Reid KE, Olsson N, Schlosser J, Peng F, Lund ST. An optimized grapevine RNA isolation procedure and statistical determination of reference genes for real-time RT-PCR during berry development. BMC Plant Biol 2006; 6: 27.

53 Livak KJ, Schmittgen TD. Analysis of relative gene expression data using real-time quantitative $P C R$ and the $2 \wedge(-\Delta \Delta C T)$ method. Methods 2001; 25: 402-408

54 Grimplet J, Adam-Blondon A-F, Bert P-F, Bitz O, Cantu D, Davies C et al. The grapevine gene nomenclature system. BMC Genomics 2014; 15: 1077

55 Vilella AJ, Severin J, Ureta-Vidal A, Heng L, Durbin R, Birney E. EnsemblCompara GeneTrees: Complete, duplication-aware phylogenetic trees in vertebrates. Genome Res 2009; 19: 327-335.

56 Rambaldi D, Ciccarelli FD. FancyGene: Dynamic visualization of gene structures and protein domain architectures on genomic loci. Bioinformatics 2009; 25: 2281-2282.

57 Thornton JW, Desalle R. Gene family evolution and homology: genomics meets phylogenetics. Annu Rev Genomics Hum Genet 2000; 1: 41-73.
58 Zhang J. Evolution by gene duplication: An update. Trends Ecol Evol 2003; 18: 292-298.

59 Wang H-W, Zhang B, Hao Y-J, Huang J, Tian A-G, Liao Y et al. The soybean Doftype transcription factor genes, GmDof4 and GmDof11, enhance lipid content in the seeds of transgenic Arabidopsis plants. Plant J 2007; 52: 716-729.

60 Doligez A, Bertrand Y, Farnos M, Grolier M, Romieu C, Esnault F et al. New stable QTLs for berry weight do not colocalize with QTLs for seed traits in cultivated grapevine (Vitis vinifera L.). BMC Plant Biol 2013; 13: 217.

61 Kennedy JA, Troup GJ, Pilbrow JR, Hutton DR, Hewitt D, Hunter CR et al. Development of seed polyphenols in berries from Vitis vinifera L. cv. Shiraz. Aust J Grape Wine Res 2000; 6: 244-254.

62 Skirycz A, Jozefczuk S, Stobiecki M, Muth D, Zanor MI, Witt I et al. Transcription factor AtDOF4.2 affects phenylpropanoid metabolism in Arabidopsis thaliana. New Phytol 2007; 175: 425-438.

This work is licensed under a Creative Commons Attribution 4.0 International License. The images or other third party material in this article are included in the article's Creative Commons license, unless indicated otherwise in the credit line; if the material is not included under the Creative Commons license, users will need to obtain permission from the license holder to reproduce the material. To view a copy of this license, visit http://creativecommons.org/licenses/ by/4.0/

(c) The Author(s) 2016

Supplementary Information for this article can be found on the Horticulture Research website (http://www.nature.com/hortres) 